\title{
An energetics-based honeybee nectar-foraging model used to assess the potential for landscape-level pesticide exposure dilution
}

Johannes M Baveco ${ }^{\text {Corresp., }}{ }^{1}$ ， Andreas Focks ${ }^{1}$ ， Dick Belgers ${ }^{1}$ ， Jozef JM van der Steen ${ }^{2}$ ， Jos JTI Boesten ${ }^{1}$, Ivo Roessink ${ }^{1}$

${ }^{1}$ Alterra, Wageningen University and Research Center, Wageningen, The Netherlands

2 Plant Research International, Wageningen University and Research Center, Wageningen, The Netherlands

Corresponding Author: Johannes M Baveco

Email address: hans.baveco@wur.nl

Estimating the exposure of honeybees to pesticides on a landscape scale requires models of their spatial foraging behaviour. For this purpose, we developed a mechanistic, energetics-based model for a single day of nectar foraging in complex landscape mosaics. Net energetic efficiency determined resource patch choice. In one version of the model a single optimal patch was selected each hour. In another version, recruitment of foragers was simulated and several patches could be exploited simultaneously. Resource availability changed during the day due to depletion and/or intrinsic properties of the resource (anthesis). The model accounted for the impact of patch distance and size, resource depletion and replenishment, competition with other nectar foragers, and seasonal and diurnal patterns in availability of nectar-providing crops and wild flowers. From the model we derived simple rules for resource patch selection, e.g., for landscapes with mass-flowering crops only, net energetic efficiency would be proportional to the ratio of the energetic content of the nectar divided by distance to the hive. We also determined maximum distances at which resources like oilseed rape and clover were still energetically attractive.

We used the model to assess the potential for pesticide exposure dilution in landscapes of different composition and complexity. Dilution means a lower concentration in nectar arriving at the hive compared to the concentration in nectar at a treated field and can result from foraging effort being diverted away from treated fields. Applying the model for all possible hive locations over a large area, distributions of dilution factors were obtained that were characterised by their 90 -percentile value.

For an area for which detailed spatial data on crops and off-field semi-natural habitats were available, we tested three landscape management scenarios that were expected to lead to exposure dilution: providing alternative resources than the target crop (oilseed 
rape) in the form of i) other untreated crop fields, ii) flower strips of different widths at field edges (off-crop in-field resources), and iii) resources on off-field (semi-natural) habitats. For both model versions significant dilution occurred only when alternative resource patches were equal or more attractive than oilseed rape, nearby and numerous and only in case of flower strips and off-field habitats. On an area-base, flower strips were more than one order of magnitude more effective than off-field habitats, the main reason being that flower strips had an optimal location.

The two model versions differed in the predicted number of resource patches exploited over the day, but mainly in landscapes with numerous small resource patches. In landscapes consisting of few large resource patches (crop fields) both versions predicted the use of a small number of patches. 


\section{AUTHORS}

Johannes M. Baveco, Alterra, Wageningen University \& Research Center, Wageningen, The Netherlands

Andreas Focks, Alterra, Wageningen University \& Research Center, Wageningen, The Netherlands

Dick Belgers, Alterra, Wageningen University \& Research Center, Wageningen, The Netherlands

Jozef J.M. van der Steen, Plant Research International, Wageningen University \& Research Center, Wageningen, The Netherlands

Jos J.T.I. Boesten, Alterra, Wageningen University \& Research Center, Wageningen, The Netherlands

Ivo Roessink, Alterra, Wageningen University \& Research Center, Wageningen, The Netherlands

Corresponding author:

Johannes M. Baveco,

Alterra, Wageningen UR,

PO Box 47, 6700 AA Wageningen, The Netherlands

Tel. +31317486095

Email: hans.baveco@,wur.nl 
1

2 There is serious concern about the widespread decline of pollinators in the agricultural landscape

3 (Potts et al. 2010; Vanbergen et al. 2013). Agricultural intensification leading to increased stress

4 from pesticides and lack of floral resources, is probably the main cause (Goulson et al. 2015).

5 Honeybees, by exploiting mass-flowering crops, operating over long distances, and by being

6 carefully managed by beekeepers, can be considered to be relatively insensitive to the

7 disappearance and deterioration of semi-natural elements providing food and nesting

8 opportunities (Ricketts et al. 2008; Sponsler \& Johnson 2015; Winfree et al. 2009). Their

9 dependence on cropland for nectar and pollen acquisition, however, leads to high potential exposure to pesticides (Krupke et al. 2012).

Understanding to what extent pesticides may affect honeybees requires as an essential first step the quantification of their potential exposure. Exposure at the hive is the outcome of foraging in the landscape surrounding the hive, as concentrations of pesticides in nectar and pollen brought by foraging bees will depend on the provenance of these food items, and thus on choice of forage and foraging locations (Garbuzov et al. 2015). It is well known that honeybees may forage over long distances (Beekman \& Ratnieks 2000), up to approximately $10 \mathrm{~km}$ away from the hive. However, when sufficient high quality resources are available nearby, most foraging will take place within a few kilometres (Couvillon et al. 2015; Couvillon et al. 2014b).

Models considering foraging in complex, heterogeneous landscapes with multiple resources to choose between, can be used to predict exposure concentrations at the bee hive. Such models may also serve as tools to test landscape management measures aimed at lowering exposure risk (mitigation). Of the numerous honeybee models reviewed in (Becher et al. 2013; Schmickl \& Crailsheim 2007) however, none were developed to address landscape-level foraging and its consequences for pesticide exposure.

We extend and adapt the energetics-based modelling approach of (Cresswell et al. 2000; Dukas \& Edelstein-Keshet 1998; Schmid-Hempel et al. 1985) to deal with foraging for nectar on a large spatial scale, in heterogeneous landscapes with multiple resources including mass-flowering crops and wild flowers in semi-natural habitats. The model simulations can use real world geographical information system (GIS) data at regional to national scales. Our model predicts 
resource patch selection during the day, taking into account resource depletion. It translates this selection into pesticide concentration in nectar arriving at the hive, when some patches are fields treated with pesticides or off-field habitats exposed to spray-drift. With the model we explore under which conditions the presence of multiple resources can divert foraging effort away from pesticide-treated fields and lead to dilution of exposure: a possible explanation of lower observed "field-realistic" rates of exposure compared to laboratory studies (Carreck \& Ratnieksi 2014).

We finally explore whether landscape management aimed at pollinator conservation may have the additional benefit of lowering the exposure of honeybees to pesticides. Three hypothetical scenarios were tested for their impact on exposure dilution, through the presence of attractive alternative crop fields, flowers strips (off-crop in-field resources), or attractive resources on offfield habitats.

\section{MATERIAL \& METHODS}

\section{Energetics-based foraging model}

The model simulates honeybee nectar foraging over a single day in hourly time steps. The model ignores in-hive colony dynamics and assumes the colony to have limited to perfect knowledge of available resources and to adapt quickly to environmental fluctuations in food conditions (Beekman \& Bin Lew 2008; Beekman et al. 2003). Resource patch selection is based on net energetic efficiency. We differentiate between two basic model versions. In the "single-optimal" (SO) version of the model we assume that the colony's self-organization is fast enough to effectively focus on the single most-profitable food patch each hour. In case of two or more approximately equally profitable sources, mechanisms should be present that reinforce the use of only one of them (e.g., symmetry breaking and cross inhibition (de Vries \& Biesmeijer 2002). In the "recruitment-limited" (RL) version we acknowledge that the rate of recruitment of foragers for a resource limits the speed with which the system can adjust to dynamic resources (Seeley \& Visscher 1988). As a consequence, in this version several resource patches with a high net energetic efficiency can be exploited simultaneously, as suggested by field observations 
58 (Beekman et al. 2004; Visscher \& Seeley 1982). In the SO-model this may occur when resource availability changes during the day, depending on the anthesis of flowers and/or due to resource depletion. In the RL-model, simultaneous exploitation of multiple patches during the day is the rule.

The model predicts for a hive at a specific location the set of resource patches used during a single day as well as several quantities that can be compared to field observations, e.g. the amount of sugar arriving at the hive, exploited nectar sources weighted by the amount of collected sugar in each, or the distribution of foraging distances. Alternatively, it allows for the quantification of impact of the hive population on a crop, e.g., as the number of flower visits per unit area, per flower or per patch.

\section{Landscape and Resources}

Foraging takes place in a landscape consisting of multiple resource patches. Each resource patch is assumed to be internally homogeneous and to contain a single resource. Resource patches may be fields with mass-flowering crops providing nectar or semi-natural habitats characterised by a dominant nectar-providing species.

Resources have a dynamic density of nectar-providing flowers $F\left(\mathrm{~m}^{-2}\right)$. In absence of nectar consumption, $F$ remains $F_{0}$. The density of open flowers is the product of plant density and the number of open flowers per plant. Resources are characterised by the average amount of nectar $g$ (mg) a honeybee can obtain from a flower, and by the typical sugar content of its nectar.

\section{Resource patch selection}

Being a central-place forager, a honeybee foraging trip comprises the travel back and forth between hive and resource patch, and the searching for and handling of flowers in the patch. Within the field, floral resources are collected assuming a type II functional response (Holling 1959), so the number of flowers with nectar that are visited per time unit (s) amounts to 
84 (eq. 1) $\quad f=\frac{a F}{1+a h F}$

85 with $a$ the attack rate $\left(\mathrm{m}^{2} \mathrm{~s}^{-1}\right)$ and $h$ the handling time per flower $(\mathrm{s})$. The rate of nectar collection

$86\left(\mathrm{mg} \mathrm{s}^{-1}\right)$ is given by $f g$. The time $t_{L}$ it takes to collect nectar to full capacity is

87

88

100

101

102

103

104

105

106

$$
t_{L}=\frac{\gamma}{f g}
$$

where the capacity is given by $\gamma(\mathrm{mg})$. Note that unlike e.g., (Schmid-Hempel et al. 1985) we assume that foragers always collect a full load.

Flight time and flight costs are proportional to the distance from hive to the resource patch. The duration of a foraging trip equals the sum of travel times and the time spent at the patch:
(eq. 3)

$$
t_{\text {trip }}=2 \frac{D}{v}+\frac{\gamma}{f g}
$$

with $D$ being the distance from the hive to the field $(\mathrm{m})$, and $v$ the flight velocity $\left(\mathrm{m} \mathrm{s}^{-1}\right)$.

The energy expenditure $E E(\mathrm{~J})$ ignoring basic metabolism can be calculated as the sum of the travel costs $E E_{\text {travel }}$ (travel time * energetic costs per time unit) and the costs while loading nectar in the field $E E_{\text {field. }}$. The latter term is made up by flight costs while searching for nectar flowers and costs while sitting on the flowers extracting the nectar. The latter is ignored, as it is approximately an order of magnitude smaller than flight costs (a value of $0.0042 \mathrm{~J} \mathrm{~s}^{-1}$ was applied in (Schmid-Hempel et al. 1985)). Energy expenditure at the field thus becomes:

$$
E E_{\text {field }}=\left(t_{L}-\frac{\gamma}{g} h\right) e_{F}=\frac{\gamma e_{F}}{g a F}
$$

The equation is simplified by substituting equations 2 and 1, thus eliminating the handling time. By assuming that handling the flower doesn't take energy, $h$ does not play a role in the energetic balance. In equation 4 average flight cost $e_{F}\left(\mathrm{~J} \mathrm{~s}^{-1}\right)$ is used, as during foraging in the field the individual state changes gradually from unloaded to loaded. The value of $e_{F}$ is obtained from loaded $e_{F, L}$ and unloaded $e_{F, U}$ flight costs (Seeley 1986). The total travel costs are given by:

$$
E E_{\text {travel }}=\frac{D}{v}\left(e_{F, U}+e_{F, L}\right)=2 \frac{D}{v} e_{F}
$$

If the same route is followed back and forth, the energy costs can be averaged. 
108 The total energy expenditure $E E_{\text {total }}(\mathrm{J})$ for a foraging bout sums to:

109

(eq. 6)

$$
E E_{\text {total }}=\left(t_{L}-\frac{\gamma}{g} h\right) e_{F}+2 \frac{D}{v} e_{F}=\left(t_{L}-\frac{\gamma}{g} h+2 \frac{D}{v}\right) e_{F}=\left(\frac{\gamma}{g a F}+2 \frac{D}{v}\right) e_{F}
$$

110 The yield of a trip in terms of energy, energy intake $E I(J)$, depends on the energy content of the

111

112 (eq. 7)

$$
E I=\gamma e_{R}
$$

113

114

115

116

117

118

119

120

121

122

123

124

125

With $t_{\text {trip }}, E E$ and $E I$, the basic ingredients for a "decision making process" for a colony are specified, and costs (both in energy and time) and yields for specific foraging locations in a landscape with multiple fields can be compared. In theory, there are different currencies that might be optimized (Stephens \& Krebs 1987). For honeybees considerable effort has been invested in deciding whether the relevant currency is the net rate of energy delivery ((gaincost)/time) or the net energetic efficiency ((gain-cost)/cost). Experimental data, e.g., (Seeley 1994) but see (de Vries \& Biesmeijer 2002), and models fit to experimental data (SchmidHempel et al. 1985), as well as several of the other foraging models discussed by (Becher et al. 2013) indicate that net efficiency is the most appropriate currency. We therefore assume that net energetic efficiency $N E E$

$$
N E E=\frac{E I-E E_{\text {total }}}{E E_{\text {total }}}
$$

defines the attractiveness of each patch. In the SO version the patch with maximum $N E E$ is selected as the single resource patch. In the RL version the recruitment of foragers for a resource patch is explicitly modelled, following (Camazine \& Sneyd 1991; Seeley et al. 1991) and extending their model in a somewhat similar way as done by (Cox \& Myerscough 2003). In the RL-model NEE is translated into the probability of foragers abandoning resources and recruiting new foragers among the follower-bees present at the dance floor (Supplemental Information Recruitment model). 
133 Resource patches are detected primarily by scouts. Scouts make up around $10 \%$ of the

134

135

136

137

138

139

140

141

142 unemployed foragers: novice foragers and experienced foragers that have recently abandoned a depleted resource patch (Seeley 1995). Detection of an attractive resource patch may depend on patch distance and size (Dauber et al. 2010), as well as on the number of active scouts. Encounter rates may be obtained from movement models or from dispersal functions summarizing movement simulations (Heinz et al. 2007), as $a_{i}$ values: the probability that a single scout encounters patch $i$. The probability for patch $i$ being detected by at least 1 of $s$ scouts amounts to

(eq. 9)

$$
P_{i}=1-\left(1-a_{i}\right)^{s}
$$

When $P$ is set to 1 for each resource patch - as we do in the studied cases - the hive population is assumed to have perfect knowledge of its environment. This setting is appropriate when dealing with a landscape with abundant resources in the immediate surroundings of the hive (Seeley 1995).

\section{Resource acquisition}

The colony's hourly acquisition of a selected resource depends on the absolute number of foragers $n$ dedicated to this resource. For the SO-model $n$ equals the total number of foragers active in a time step. For the RL-model, $n$ is a fraction of this total number, as multiple patches may be exploited simultaneously. Foragers may make several trips to the same patch, depending on trip duration $t_{\text {trip }}$ and the time between trips $t_{U D}$ spent on unloading and dancing in the hive. Thus, the number of foraging trips per hour an individual forager can make, amounts to $b=\Delta t /$ $\left(t_{\text {trip }}+t_{U D}\right)$ with $\Delta t$ representing the time step in seconds.

Not the full load of nectar $\gamma$ collected in the patch will arrive at the hive as some of the sugar will be consumed on the way back. The amount of energy used during the return flight, $E_{c}$, is approximately $e_{L} \frac{D}{v}$. The amount of nectar arriving at the hive thus decreases to $\gamma-\frac{E_{c}}{e_{R}}$ per trip and when summed over all foragers exploiting the patch during this time step becomes:

(eq. 10) $\quad n\left(\gamma-\frac{E_{c}}{e_{R}}\right) b$ 


\section{Resource dynamics}

161 By calculating resource patch selection per hour we may account for, 1) any diurnal pattern in 162 the anthesis of flowers, 2) depletion of floral resources occurring in small resource patches, and, 163 3) diurnal patterns in the number of active foragers in the hive. For simplicity's sake we assume 164 a binary pattern in anthesis (all flowers either open or closed).

165

Resource dynamics may also result from nectar consumption by competing species or foragers from other colonies. A constant background density of competitors, $Z$, may be defined of species that exploit resources in a similar way as honeybees (same functional response). Main reason to incorporate competition is that it allows us to explore honeybee foraging in a setting of quickly depleting resources.

Within a day there may be also renewal of nectar resources. With a non-zero renewal rate, $r$, a fraction of the pool of visited (empty) flowers will each time step turn into nectar-providing flowers again.

We deal with resource depletion by subtracting after each time step the visited number of open flowers from the initially present number. Hourly dynamics in open flower density $F$ are given by:

(eq. 11) $\quad F_{t+1}=F_{t}-\frac{n \gamma}{g A} b-\frac{a F}{1+a h F} Z+r\left(F_{0}-F\right)$

with $A$ representing field size $\left(\mathrm{m}^{2}\right)$. A fixed number of flowers $\gamma / g$ is visited for a full load. Lowered flower density may change the $N E E$-based ranking of resource patches in the next time step.

\section{Exposure Assessment}

When the foraging model is combined with information on fields being treated with a chemical, and/or off-field habitats being exposed to spray-drift, the concentration of the chemical in the nectar brought into the hive can be estimated. It depends on the concentration of the chemical in 
185 the nectar of flowers in exploited patch $i, C_{i}$, expressed in e.g., $\mu \mathrm{g} \mathrm{mg}^{-1}$. When the chemical is 186 not metabolized together with the sugar on the way back to the hive, its total amount (mg) 187 arriving at the hive will be:

$188 \quad$ (eq. 12) $\quad \sum_{i=1}^{L} n_{i} \gamma b_{i} C_{i}$

189 The summation is made over $L$, the set of all exploited patches over all hours. The concentration 190 of the chemical in nectar at arrival no longer equals $C_{i}$ but becomes $C_{i} /\left(1-\frac{E_{c, i}}{\gamma e_{R, i}}\right)$, implying 191 enrichment. When the chemical is metabolized together with the sugar, its concentration in the 192 nectar remains the same but the amount arriving at the hive will be lower:

193

200

(eq. 13) $\quad \sum_{i=1}^{L} n_{i}\left(\gamma-\frac{E_{c, i}}{e_{R, i}}\right) b_{i} C_{i}$

From the point of view of exposure risk inside the hive, the relevant chemical concentration should be expressed on a sugar base: nectar with a low sugar content will be concentrated until a minimum sugar content is reached. The exposure on a sugar-base is obtained by dividing the total amount of the chemical $(\mu \mathrm{g})$ entering the hive by the total amount of sugar entering the hive (mg). Without metabolization of the chemical (with enrichment) we obtain as sugar-based daily averaged concentration of the chemical $\left(\mu \mathrm{g} \mathrm{mg}^{-1}\right)$ entering the hive:

(eq. 14)

$$
X_{e}=\frac{\sum_{i=1}^{L} n_{i} \gamma b_{i} C_{i}}{\sum_{i=1}^{L} n_{i} \frac{\left(\gamma e_{R, i}-E_{c, i}\right)}{e_{S U G A R}} b_{i}}
$$

When the chemical is metabolized with the sugar (no enrichment) we obtain:

(eq. 15)

$$
X_{m}=\frac{\sum_{i=1}^{L} n_{i}\left(\gamma-\frac{E_{c, i}}{e_{R, i}} b_{i} C_{i}\right.}{\sum_{i=1}^{L} n_{i} \frac{\left(\gamma e_{R_{i}}-E_{c, i}\right)}{e_{S U G A R}} b_{i}}
$$

Equations 14 and 15 quantify the contribution of each selected resource patch to the sugar-based concentration of a chemical ( $\mu \mathrm{g}$ chemical $\mathrm{mg}^{-1}$ sugar) entering the hive. Using this information together with the calculated amount of sugar having this concentration we can construct detailed distributions quantifying the relative composition of the nectar entering a particular hive, in terms of sugar-based concentrations. 
Dilution

210 Exposure dilution is caused by foraging on other resources than a treated resource when these

211 other resources are less or not contaminated. Dilution is defined relative to a reference

212 concentration in treated resources, e.g., the sugar-based concentration $X_{P}$ in the nectar of a

213 treated field with resource $R$ :

214 (eq. 16)

$$
X_{P}=C_{P} \frac{e_{S U G A R}}{e_{R}}
$$

215

216

217

218

219

220

221

222

223

224

228

229

230

where $C_{P}$ refers to the concentration (on a wet-weight base) in nectar on the treated field, often referred to as the predicted environmental concentration $(P E C)$ resulting from a certain application rate of the chemical. It is multiplied by $e_{S U G A R} / e_{R}$ to obtain the sugar-based concentration. Dilution factors follow from actual $X_{m}$ or $X_{e}$ as:

(eq. 17)

$$
\varphi_{m}=X_{m} / X_{P} \text { and } \varphi_{e}=X_{e} / X_{P}
$$

With a patch-specific $C$ we can represent variability in the applied dose for the treated crop, deal with a substance that is applied on different crops with different doses, and include patches representing off-crop or off-field habitats that are exposed through spray drift only.

For an agricultural landscape containing fields with a target crop as the only resources, equations 14 and 15 can be further simplified. With a single resource $e_{R, i}$ equals $e_{R}$ and when application of a pesticide on the target crop leads to a constant concentration $C_{P}$ in the nectar, $C_{i}$ equals $C_{P}$. Dilution obtained applying equation (14) then simplifies to:

(eq. 18)

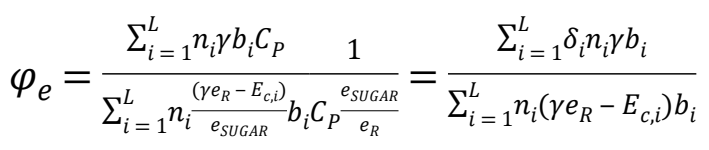

with $\delta_{i}=1$ in case the field selected in time step $i$ is sprayed and $\delta_{i}=0$ if it is unsprayed.

Similarly, for the case with metabolization and no enrichment (Eq. 15) we obtain: 
231 (eq. 19)

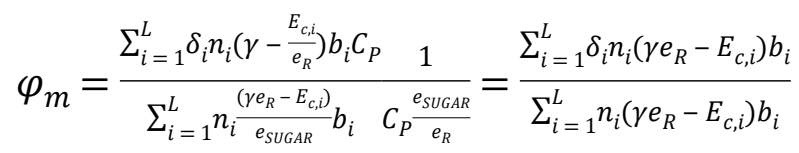

232 Equations 18 and 19 show that for this simplified case, dilution factors do not depend on $C_{P}$ but 233 simply reflect how many unsprayed fields are selected. When all selected fields are treated $\varphi_{m}$ 234 will be one, while $\varphi_{e}$ may even exceed one. Whether selected resource patches were treated or 235 not depends on the application scenarios. In a probabilistic approach, with $p$ representing the probability for a field of being treated, dilution will on average approximate $p$.

\section{$90^{\text {th }}$ percentiles}

239

240

241

242

243

244

In an exposure assessment the model will be applied for all possible locations of an apiary within the area of use of a substance. Following recent guidance (EFSA 2013), all sites at edges of target crop fields are considered to be potential locations and the target crop patch adjacent to the hive location is always assumed to be sprayed or treated. From application of the model at each site, we construct cumulative distributions of exposure or dilution from which statistical descriptors can be obtained, e.g., the $90^{\text {th }}$ percentile that quantifies the dilution obtained at $90 \%$ of all sites, or the exposure that is exceeded at $10 \%$ of the sites.

\section{Model Analysis \& Application}

\section{Energetics-based model}

From the energetics-based model (Eq. 6 and 7) we derived thresholds for the exploitation of resources depending on distance and resource characteristics, as done by (Cresswell et al. 2000). For resources at equal distance we derived a simple equation to compare their attractiveness. For landscapes consisting of only large-scale crop fields, we derived rules of thumb for the selection of foraging locations. 
256

257

258

259

260

261

262

263

264

265

266

267

268

269

270

271

272

273

274

275

276

277

278

279

280

281

282

283

284

285

\section{Landscape representation}

When it is assumed that nectar is collected from all resources in the landscape proportional to their attractiveness (EFSA 2013), exposure dilution is always to be expected in landscape mosaics with multiple resource patches. In the RL-model dilution is more likely than in the SOmodel. To explore whether landscape-level exposure mitigation can be effective, we tested for both models three scenarios, all based on the idea that by providing alternative floral patches foraging effort can be diverted away from exposed patches. Construction of flower strips and pollinator-friendly management of semi-natural habitats are such measurements that may contribute to the persistence of pollinator populations in the agricultural landscape (Garibaldi et al. 2014; Haaland et al. 2011; Wratten et al. 2012). Also the presence of different (early and late) mass-flowering crops has been suggested to enhance pollinator density (Riedinger et al. 2014).

We parameterized the model for two flower species (Table 2), oil-seed rape (Brassica napus, OSR) as a common attractive mass-flowering crop, and white clover (Trifolium repens) being an important floral resource, common along roadsides and field margins (Sponsler \& Johnson 2015). Patches with these resources provided the building blocks of the landscapes tested in the scenarios. In all scenarios OSR represented the target crop. From the model it followed that for any of the scenarios to be effective the alternative resource had to be equally or more attractive (larger NEE at equal distance) than the OSR field. For the scenario study, using a hypothetical landscape, we selected for OSR a conservative and for clover an optimistic estimate of the relevant coefficients (Table 2 and Supplemental Information) and checked whether this condition was met (see results section). In real landscape clearly more resource types will be present, in a range of densities. To understand the impact of some of this variability we varied flower densities and sugar content over a range of values.

We defined landscape structure from geographic data for the northern part of Flevoland (The Netherlands). Geo-referenced data for crops were obtained from the land-use database LGN6 (grid-based, $25 \mathrm{~m}$ resolution) (Hazeu et al. 2011). Data on the presence of off-field habitats (road side verges, ditch sides, shores, other semi-natural elements) were obtained from the vectorbased dataset TOP10NL (PDOK 2015). We assumed that fields of LGN6 category "other crops" represented OSR. For the "Alternative Fields" scenario, fields of another (randomly chosen)

LGN6 category represented the alternative mass-flowering crop (Fig. 1). For the "Off-field 
286

287

288

289

290

291

292

293

294

295

296

297

298

299

300

301

302

303

Habitats" scenario, off-field habitats were used as specified in the spatial data set. All sites (cells in the $25 \mathrm{~m}$ grid) that bordered target crop fields were considered as potential beehive locations (Supplemental Figure S1). To avoid excessive computing times the model was finally run on a random selection of $10 \%$ of these sites (identical for all scenarios and runs). Tested landscapes contained 1207 fields with OSR (7057 ha). The alternative crop was present on 454 fields (3375 ha). Off-field habitat patches were small and numerous: 58137 patches comprising 3410 ha.

We assumed all resources to have open flowers during the whole day, a foraging day of 10 hours, no competitors being present $(Z=0)$, no renewal of resources $(r=0)$ and a constant number of active foragers (Table 1 and 2). By definition, sites were next to a large resource patch. Therefore, considering only resources within $2 \mathrm{~km}$ distance $\left(D_{\max }=2000\right)$ and assuming perfect knowledge of their presence ( $P=1$ for each resource patch) seemed reasonable. For simplicity's sake we assumed that the hypothetical pesticide was metabolized with the consumed sugar on the way back to the hive, so without dilution the dilution factor would imply be one. The nearby crop field was assumed to be always treated with the pesticide.

\section{Exposure mitigation}

i) Alternative Fields: Dilution can result from the presence of attractive but untreated fields, either with the target crop or with alternative attractive (mass-flowering) crops. For crop fields other than the one nearest to the hive, treatment occurred following a probability $p$. The average dilution factor for a site was then calculated from 100 different random realizations of the series of treated fields. The analysis was done for a range of values for $p$ ( 0 to 1 , with an interval of 0.1 ). A variant of this mechanism would be the presence of fields with another, attractive, crop type. We tested this by defining another common crop in Flevoland as a hypothetical alternative mass-flowering crop, largely identical to oil-seed rape (Fig. 1). The energetic attractiveness of this crop was manipulated to range from less to more attractive than OSR, by adjusting its sugar content $(0.8,0.9,1,1.1$ and 1.2 times OSR sugar content). Thus, the alternative crop could also represent another OSR variety.

ii) Flower Strips: Dilution can result from the presence of highly attractive flower strips around target crop fields. This was simulated by adding to each target crop field up to 4 flower strips at 
315 its edges, each of length $1 / 4$ of field perimeter. Strips contained white clover (Trifolium repens,

316 table 2) in densities comparable to clover fields. Presence of each strip was randomly set, with a

317 probability $p$ ( 0 to 1 , with an interval of 0.1 ). The area of a flower strip was set to a prescribed

318 width $w$ multiplied by strip length. Width values of 1, 2, 5 and $10 \mathrm{~m}$ were tested. The area

319 contained in the strips was subtracted from the crop field area. Thus, strips were strictly in-field

320 off-crop habitats.

321

322

323

324

325

326

327

328

329

330

331

332

333

334

335

336

337

338 339

340

iii) Off-field Habitats: dilution can result from the presence of off-field semi-natural habitats when these are managed in an appropriate way. We tested this using geo-data for fields (as above) with OSR and for semi-natural elements (Fig. 1). We assumed a single common flower species (white clover) to be representative for all off-field habitats. As the presence and size of these habitats were fixed and defined by the geo-data, we tested their impact on dilution for a range of resource quality values. Open flower density determined quality and was set to low, medium or high value $(0.5,1$ or 1.5 times clover field density). The three quality levels were tested for a range of values for $p$, here representing the probability that an off-field element was considered a nectar resource patch.

\section{RESULTS}

Energetics-based model

From the energetics-based model (Eq. 6 and 7) we derived thresholds for the exploitation of resources depending on distance to the resource patch and other resource characteristics. The energy balance $(E I-E E)$ for a foraging trip has to be positive (Dukas \& Edelstein-Keshet 1998), leading to the condition:

(eq. 20)

$$
\gamma e_{R}-\left(\frac{\gamma}{g a F}+2 \frac{D}{v}\right) e_{F}>0
$$

The threshold distance at which a resource patch cannot be exploited any more (Fig. S2) amounts to

(eq. 21)

$$
D_{T}=\frac{v \gamma}{2}\left[\frac{e_{R}}{e_{F}}-\frac{1}{g}\left(\frac{1}{f}-h\right)\right]=\frac{v \gamma}{2}\left[\frac{e_{R}}{e_{F}}-\frac{1}{g a F}\right]
$$


341 Resource patch selection based on net energetic efficiency implies selecting the patch for which

342 the ratio between energy intake and expenditure $E I / E E$ is largest:

343 (eq. 22)

$$
\frac{E I}{E E}=\frac{\gamma e_{R}}{\left(\frac{\gamma}{g a F}+2 \frac{D}{v}\right) e_{F}}
$$

344 This can be rewritten to:

345 (eq. 22a)

$$
\frac{E I}{E E}=\frac{g a F}{\left(1+\frac{2 D}{\gamma v} g a F\right)} e_{R} / e_{F}
$$

346 Or, substituting $\frac{2 D}{\gamma v}$ by constant $c$ for patches at equal distance:

347 (eq. 22b)

$$
\frac{E I}{E E}=\frac{g a F}{(1+c g a F)} e_{R} / e_{F}
$$

348 Figure 2 shows how this ratio depends on resource characteristics $g a F$, the nectar acquisition rate. At equal distance, $E I / E E$ scales linearly with energetic content $e_{R}$ and asymptotically with $g a F$. In a mass-flowering case, a further increase of $g a F$ will not increase $E I / E E$ much. For a "sparse-flowering" resource to compete in attractiveness with a mass-flowering crop, its energy content or flower density needs to be considerably higher.

353

354

355

356

357

358

359

360

361
An 'extreme' case results from landscapes consisting entirely of fields with mass-flowering crops. There, open flower density $F$ will be very large, and the functional response $f$ will approach $1 / h$. As a consequence, the energy spent in the field $E E_{\text {field }}$ will be approaching zero and $E E$ will thus be determined mostly by $E E_{\text {travel }}$. The threshold distance (Eq. 21) will simplify to a linear relationship with $e_{R}$, with steepness independent of other crop properties; the honeybee constants are given between brackets:

(eq. 23)

$$
D_{T} \approx\left[\frac{\gamma v}{2 e_{F}}\right] e_{R}
$$

Maximising the net energetic efficiency in the mass-flowering case means maximising

(eq. 24)

$$
\frac{E I}{E E} \approx\left[\frac{\gamma v}{2 e_{F}}\right] \frac{e_{R}}{D}
$$


362 For fields at equal distance, the selected field will thus be the one with the highest energy content

$363 e_{R}\left(\mathrm{~J} \mathrm{mg}^{-1}\right.$ nectar $)$.

Landscape scenarios

366

367

368

369

370

371

372

373

374

375

376

377

378

379

380

381

382

383

384

385

386

387

388

Cumulative distributions of dilution factors were discontinuous for the SO-model, with many sites experiencing no dilution at all, others having no exposure and some experiencing limited dilution (Fig. S3 to Fig. S6)). For the RL-model the distributions were continuous, with few sites being without exposure or without dilution. All distributions were summarized by their 10-, 50and 90-percentiles (Fig. S6). For all scenarios the number of resource patches exploited over the day were obtained as well, as a characteristic output of the foraging model. For the RL-model, this referred to the number of resource patches accounting for $90 \%$ of the sugar brought to the hive.

\section{"Alternative Fields"}

In this scenario the number of resource patches and resource area were constant. In two subscenarios either only OSR fields or OSR fields plus fields with a similar crop of different quality (sugar content) were present.

When fields with OSR were the only resource patches, a smaller (SO-model) or larger (RLmodel) fraction of the sites showed considerable dilution when a fraction of these fields were not sprayed (Supplemental Figures S3). Even when none of the fields besides the nearest were treated there was no site completely without exposure, indicating that the nearest (by definition treated) field was always included in the set of resource patches exploited during the day. For the RL-model, there was always some dilution, especially when the fields selected besides the nearest field had a high probability of not being sprayed.

In the presence of an alternative untreated crop there was a fraction of sites for which an alternative crop field was included in the set of exploited patches, resulting in dilution (Fig. S4).

For the SO-model, these sites had no exposure at all, implying that this alternative crop field 
389

390

391

392

393

394

395

396

397

398

399

400

401

402

403

404

405

406

407

408

409

410

411

412

413

414

415

416

constituted the single patch exploited during the day. Depletion played no role on large fields and either the nearest OSR field or an alternative crop field was selected and remained optimal during the whole day. For the RL-model, there was always some dilution, but there were no sites completely without exposure as the nearby OSR field was always part of the exploited patches set.

The number of exploited patches over the day was on average small: one for the SO-model and approximately 3 to 4 for the RL-model (Fig. S7). Thus the resource patch chosen at the beginning of the day usually stayed the optimal one (SO-model) during the day, as depletion was unlikely on (large) fields. In this structurally simple landscape there were always only a few nearby attractive fields that were exploited simultaneously in the RL-model. With increased sugar content the distance over which the alternative crop was energetically attractive increased and thus the number of exploited patches became larger (RL-model).

\section{"Flower Strips"}

In this scenario the number of resource patches increased with $p$, the probability of a flower strip being present at a side of a OSR field. Total resource area remained constant, but the ratio between the two resource types changed with $w$, strip width, as strips were located in-field.

For both models the fraction of sites with considerable dilution increased profoundly with $p$ (Fig. 3a and Fig. S5). For the SO-model (Fig. S5 and S6) increasing the width of strips increased the dilution reached by $90 \%$ of the sites. Wider strips could keep up a higher $N E E$ than the OSR field over a longer time as depletion in the wider strips was less likely. For the RL-model, impact of width was negligible: depletion was unlikely as foraging effort was already divided over multiple patches representing a relatively large area (including the OSR field).

For the RL-model the number of exploited patches (Fig. 4b) increased linearly with $p$ : more strips being present in the neighbourhood implied more patches being used. For the SO-model (Fig. 4a) only with narrow strips the number of exploited patches per day increased with $p$. Narrow strips were quickly depleted and when more strips were present in the neighbourhood another strip could become the optimal patch of the next hour. Wider strips implied less 
417 depletion and strips being selected as optimal at the beginning of the day had a higher chance of

418 remaining the single optimal patch during the day.

419

420

\section{"Off-field Habitats"}

421 In this scenario the number of resource patches and resource area increased with $p$, the

422 probability of an off-field habitat patch being managed for nectar resources of different quality

423 (clover open flower density).

424 Presence of high quality off-field resource patches affected the distributions of dilution factors in 425 a similar way as narrow flower strips (Fig. 3bcd, Supplemental Figures S6). The dilution factor

426 cumulative distribution was very sensitive to off-field resource quality. With medium quality less 427 dilution was reached at all sites and for low quality hardly any off-field resource patches were 428 selected at all.

429 The number of exploited patches showed a similar pattern as in the flower strips scenario (Fig. $4304 \mathrm{~cd}$ ). For the SO-model with high and medium quality off-field habitats the exploited patch 431 number increased with $p$ to 4 while for low quality it remained constant (1). For the RL-model 432 exploited patch number increased linearly with $p$ reaching a very high level (average of 75) with 433 high quality off-field habitats.

Comparing scenarios

436

The "Flower Strips" scenario and "Off-field Habitats" medium quality scenario were similar as both created the same type of new resource patches, in-field versus off-field. We compared their effectivity by plotting the dilution percentiles against the total area covered by the patches. The "Flower Strips" scenario with narrow strips $(1 \mathrm{~m})$ appeared roughly 35 times as efficient as the “Off-field habitat" scenario (Fig. 5) as flower strips were in-field resource patches located always near the considered bee hive. Off-field resource patches on the other hand were not guaranteed to be sufficiently close to a target crop field and the bee hive site to have an impact. 
444

445

446

\section{DISCUSSION \& CONCLUSIONS}

\section{Conceptual model}

In the model the selection of a resource patch was based on energetic efficiency. This implies that the duration of all phases of a foraging trip, including handling time of the flowers, did not affect patch choice. Duration did however determine the number of trips that could be made, and thus the colony's rate of resource acquisition and of resource depletion. In a similar way, the size of a resource patch had no direct effect on its attractiveness, only indirectly through faster depletion of smaller patches. A smaller patch may also have a larger probability of remaining undetected (Dauber et al. 2010). With a fixed number of foragers exploiting a single patch (SOmodel) or being distributed over a small set of patches (RL-model) the model predicted a decreasing flower visiting rate with patch size. This seems in accordance with findings of Goulson (2000).

The maximum distance at which nectar-providing resource patches can be exploited is given by Equation 21. Based on the values of Table 1 and 2 the distances estimated for OSR and clover fields, 8.1 and $12.7 \mathrm{~km}$, respectively, are well within the range of maximum observed foraging distances. For these mass-flowering crops, the threshold distance is mainly defined by the energy content of their nectar (Eq. 23). For natural elements with sparser flower distributions than found on clover fields, maximum distances are likely lower (Fig. 2), as searching times will be higher, increasing the energetic costs of traveling between flowers.

\section{Resource patch selection}

On a landscape level, the choice between resource patches depended on the relative value of their energetic efficiency. The attractiveness of different resources at different distances can be compared with each other using equation 22, hence exposure mitigation strategies based on the provision of alternative uncontaminated resource patches can be evaluated based on our model. These alternative patches need to provide resources that are at least equally (RL-model) or more (SO-model) attractive. 
471 The structure of the landscape determined to a large extent the number of daily exploited

472 resource patches. In a coarse-grained landscape with few large resource patches of mass-

473 flowering crops the use of a single to a few patches was predicted by the SO- and the RL-model,

474 respectively. In landscapes with many small resource patches, however, results differed

475 considerably between the two model versions. The SO-model could in theory predict up to 10

476 different patches to be exploited, one for each hour. However, this was not observed, as

477 switching each hour to a new resource would require strong resource depletion to take place. The

478 RL-model could for the same landscape predict up to 75 patches accounting for in total $90 \%$ of

479 the daily nectar collection. Assuming competition with other nectar-foraging species or other

480 colonies (Fig. S11 compared to S8) indeed increased the number of exploited patches for the

481 SO-model because of earlier depletion of the selected patch, while it decreased for the RL-model

482 presumably because the total set of attractive patches over the day became smaller.

483 For a colony of feral honeybees in temperate deciduous forest, on average $9.7( \pm 4.9)$ resource

484 patches (pollen and nectar) accounted for $90 \%$ of the daily foraging activity (Visscher \& Seeley

485 1982). For two colonies of African honeybees in the Okavango River Delta, (Schneider 1989)

486 found that $16.2( \pm 9.6)$ and $17.5( \pm 5.9)$ sites/day accounted for $90 \%$ of the daily foraging activity

487 on pollen and nectar. In both studies a continuous day-to-day redistribution of foragers over

488 patches was observed, with many more patches being present than used each day. Beekman and

489 colleagues (2004) found for two large and two small colonies of honeybees in an urban

490 environment on average 15.8 and 15.0 patches being used per day (nectar and pollen),

491 respectively.

492 These observations indicate that less optimization might happen than assumed in the SO-model,

493 but also that less dilution of foraging effort is occurring than predicted by the RL-model for

494 landscapes with many resource patches.

Landscape level exposure dilution

497 In current agro-ecosystems few high quality off-field resource patches will be available to honey bees (De la Rúa et al. 2009). The energetic model predicts that mass-flowering crops will be 499 selected even when these are located at a large distance from the hive. Observations indeed 
500 indicate that in the period during which mass-flowering crops are available, these crops 501 constitute the predominant nectar source (Requier et al. 2015). The off-field resource patches 502 that are present will under these conditions experience pollinator dilution (Holzschuh et al. 503 2011), i.e. reduced wild plant pollination because OSR fields are preferred. Depletion is not 504 likely to play a large role in these crop-dominated systems, as most crop fields are large and

505

506 507 508

flower densities in the saturating range of the functional response. The model thus suggests that in general there will be little potential for dilution of exposure in such landscapes, because the only resource that can be as attractive as a mass-flowering crop is another potentially sprayed mass-flowering crop.

We tested three mechanisms for exposure reduction at landscape scales. Starting from the worstcase assumption that the nearest target crop field was always treated, all tests indicated that there were only chances for dilution when uncontaminated patches were equally or more attractive than the target crop, nearby, numerous and of sufficient size. In a landscape with only crop fields providing resources, dilution appeared unlikely because the probability of having a more attractive uncontaminated field in the neighbourhood was simply too small, even though this neighbourhood became extended when sugar content was higher. Flower strips had the highest potential for dilution at the beehive: when all target crop fields were surrounded by some flowers strips the 90-percentile dilution factor became small for almost all sites. Off-field habitat patches were more than one order of magnitude less efficient compared to flower strips: around 30 times as much managed area was needed to achieve the same dilution. The advantage of flower strips was that they were located within the target crop field, so they easily meet the small distance condition. On the other hand, such a location in a target crop field may pose additional exposure risk when crop treatment causes also exposure in the strips. With spray drift, effectiveness in attracting foragers may trade off against higher exposure risk for flower strips, and it might be possible to achieve a higher dilution for off-field patches that are carefully chosen with respect to their location relative to crop fields: close enough to attract foragers, but far enough to avoid contamination.

\section{Model complexity and limitations of the predictions}

The current application of the developed model was not meant to deliver accurate predictions of absolute real-world nectar concentrations at the beehive. The purpose was instead to calculate the 
530

531

532

533

534

535

536

537

538

539

540

541

542

543

544

545

546

547

548

549

550

551

552

553

554

555

556

557

558

dilution factor as a relative measure of the effect of alternative nectar sources on worst-case nectar concentration estimates. The model equations as presented in this manuscript are in principle realistic enough to deliver absolute exposure estimates at landscape levels. This could, however, only be achieved with using more realistic landscape information of higher quality.

For the foraging model, realism means that the presence and status of all relevant sources of nectar in the landscape need to be known. When these data were available, predicted foraging locations could be compared to empirical data as for example obtained from dance analyses (Couvillon et al. 2014a). Regarding exposure, realism means that all applications of a pesticide in the considered region have to be taken into account, and the contamination not only of the treated crops but also of in-field flower strips and off-field patches of natural habitats providing nectar flowers has to be considered. Such real world spatially-explicit exposure would be complex, and dependent on application patterns and the fate pesticides in the field. For instance, exposure to systemic insecticides was reported to be even larger in wildflower patches than in the actual crop on which they were applied (Botias et al. 2015).

Colony dynamics as e.g. explicitly simulated in the BEEHAVE model (Becher et al. 2014) were not considered. Focussing on exposure resulting from foraging allowed us instead to avoid the complexity and the high data requirements of full colony models and made it feasible to apply the model in assessments at large spatial scales. The model dealt with nectar foraging only, as pollen foraging follows different rules. Pollen sources may not differ as much in quality as nectar sources, and net energetic efficiency does not play a role. A colony's annual need of pollen (protein) is smaller than that of nectar (25 compared to $125 \mathrm{~kg}$ (Seeley 1985)) and the overall sugar consumption of individual bees exceeds the protein consumption about 5 times (Rortais et al. 2005). However, there are good reasons to address also exposure via pollen and to develop a landscape-level pollen foraging model to be used in combination with our nectar foraging model. For instance, systemic insecticide concentrations may be higher in pollen, pollen is consumed by nurse bees that may constitute a critical, sensitive subset of the colony population, and pollen is directly stored, unmixed, and thus potentially preserves any high doses that might occur.

We applied the energetics-based model in a theoretical analysis of mitigation options, based to a large extent on real-landscape data. The results show the potential of using the model in a large- 
559 scale risk assessment and give general insight in the type of mitigation strategy that has the 560 highest chance to be effective.

561

562

563

564

565

566

567

568

569

570

571

572

573

574

575

576

577

578

579

580

581

582

583

584

585

586

\section{REFERENCES}

Becher MA, Grimm V, Thorbek P, Horn J, Kennedy PJ, and Osborne JL. 2014. BEEHAVE: a systems model of honeybee colony dynamics and foraging to explore multifactorial causes of colony failure. Journal of Applied Ecology 51:470-482.

Becher MA, Osborne JL, Thorbek P, Kennedy PJ, and Grimm V. 2013. Towards a systems approach for understanding honeybee decline: A stocktaking and synthesis of existing models. Journal of Applied Ecology 50:868-880.

Beekman M, and Bin Lew J. 2008. Foraging in honeybees - when does it pay to dance? Behavioral Ecology 19:255-262.

Beekman M, Oldroyd BP, and Myerscough MR. 2003. Sticking to their choice - honey bee subfamilies abandon declining food sources at a slow but uniform rate. Ecological Entomology 28:233-238.

Beekman M, and Ratnieks FLW. 2000. Long-range foraging by the honey-bee, Apis mellifera L. Functional Ecology 14:490-496.

Beekman M, Sumpter DJT, Seraphides N, and Ratnieks FLW. 2004. Comparing foraging behaviour of small and large honey-bee colonies by decoding waggle dances made by foragers. Functional Ecology 18:829-835.

Botias C, David A, Horwood J, Abdul-Sada A, Nicholls E, Hill E, and Goulson D. 2015. Neonicotinoid Residues in Wildflowers, a Potential Route of Chronic Exposure for Bees. Environmental Science \& Technology 49:12731-12740.

Burdon JJ. 1983. Trifolium Repens L. Journal of Ecology 71:307-330.

Camazine S, and Sneyd J. 1991. A MODEL OF COLLECTIVE NECTAR SOURCE SELECTION BY HONEY-BEES - SELF-ORGANIZATION THROUGH SIMPLE RULES. Journal of Theoretical Biology 149:547-571. 
587

588

589

590

591

592

593

594

595

596

597

598

599

600

601

602

603

604

605

606

607

608

609

610

611

612

613

614

615

616

Carreck NL, and Ratnieksi FLW. 2014. The dose makes the poison: have "field realistic" rates of exposure of bees to neonicotinoid insecticides been overestimated in laboratory studies? Journal of Apicultural Research 53:607-614.

Couvillon M, Riddell Pearce FC, Accleton C, Fensome KA, Quah SKL, Taylor EL, and Ratnieks FLW. 2015. Honey bee foraging distance depends on month and forage type. Apidologie 46:61-70.

Couvillon MJ, Schurch R, and Ratnieks FLW. 2014a. Dancing Bees Communicate a Foraging Preference for Rural Lands in High-Level Agri-Environment Schemes. Current Biology 24:1212-1215.

Couvillon MJ, Schurch R, and Ratnieks FLW. 2014b. Waggle Dance Distances as Integrative Indicators of Seasonal Foraging Challenges. PloS one 9.

Cox MD, and Myerscough MR. 2003. A flexible model of foraging by a honey bee colony: the effects of individual behaviour on foraging success. Journal of Theoretical Biology 223:179-197.

Cresswell JE, Osborne JL, and Goulson D. 2000. An economic model of the limits to foraging range in central place foragers with numerical solutions for bumblebees. Ecological Entomology 25:249-255.

Dauber J, Biesmeijer JC, Gabriel D, Kunin WE, Lamborn E, Meyer B, Nielsen A, Potts SG, Roberts SPM, Sõber V, Settele J, Steffan-Dewenter I, Stout JC, Teder T, Tscheulin T, Vivarelli D, and Petanidou T. 2010. Effects of patch size and density on flower visitation and seed set of wild plants: a pan-European approach. Journal of Ecology 98:188-196.

De la Rúa P, Jaffé R, Dall'Olio R, Muñoz I, and Serrano J. 2009. Biodiversity, conservation and current threats to European honeybees. Apidologie 40:263-284.

de Vries H, and Biesmeijer JC. 1998. Modelling collective foraging by means of individual behaviour rules in honey-bees. Behavioral Ecology and Sociobiology 44:109-124.

de Vries H, and Biesmeijer JC. 2002. Self-organization in collective honeybee foraging: emergence of symmetry breaking, cross inhibition and equal harvest-rate distribution. Behavioral Ecology and Sociobiology 51:557-569.

Dukas R, and Edelstein-Keshet L. 1998. The Spatial Distribution of Colonial Food Provisioners. Journal of Theoretical Biology 190:121-134. 
617 EFSA. 2013. Guidance on the risk assessment of plant protection products on bees (Apis

618

619

620

621

622

623

624

625

626

627

628

629

630

631

632

633

634

635

636

637

638

639

640

641

642

643

644

645 mellifera, Bombus spp. and solitary bees). EFSA Journal 11:3295. Available online: www.efsa.europa.eu/efsajournal.

Free JB, and Nuttall PM. 1968. POLLINATION OF OILSEED RAPE (BRASSICA NAPUS) AND BEHAVIOUR OF BEES ON CROP. Journal of Agricultural Science 71:91-\&.

Garbuzov M, Couvillon MJ, Schurch R, and Ratnieks FLW. 2015. Honey bee dance decoding and pollen-load analysis show limited foraging on spring-flowering oilseed rape, a potential source of neonicotinoid contamination. Agriculture Ecosystems \& Environment 203:62-68.

Garibaldi LA, Carvalheiro LG, Leonhardt SD, Aizen MA, Blaauw BR, Isaacs R, Kuhlmann M, Kleijn D, Klein AM, Kremen C, Morandin L, Scheper J, and Winfree R. 2014. From research to action: Enhancing crop yield through wild pollinators. Frontiers in Ecology and the Environment 12:439-447.

Goodwin RM, Cox HM, Taylor MA, Evans LJ, and McBrydie HM. 2011. Number of honey bee visits required to fully pollinate white clover (Trifolium repens) seed crops in Canterbury, New Zealand. New Zealand Journal of Crop and Horticultural Science 39:7-19.

Goulson D. 2000. Why do pollinators visit proportionally fewer flowers in large patches? Oikos 91:485-492.

Goulson D, Nicholls E, Botias C, and Rotheray EL. 2015. Bee declines driven by combined stress from parasites, pesticides, and lack of flowers. Science 347:1435-+.

Haaland C, Naisbit RE, and Bersier LF. 2011. Sown wildflower strips for insect conservation: a review. Insect Conservation and Diversity 4:60-80.

Hazeu GW, Bregt A, De Wit A, and Clevers J. 2011. A Dutch multi-date land use database: identification of real and methodological changes. Internat $J$ Appl Earth Obs Geoinfo 13:682-689.

Heinz SK, Wissel C, Conradt L, and Frank K. 2007. Integrating individual movement behaviour into dispersal functions. Journal of Theoretical Biology 245:601-609.

Holling CS. 1959. Some Characteristics of Simple Types of Predation and Parasitism. The Canadian Entomologist 91:385-398. 
646

647

648

649

650

651

652

653

654

655

656

657

658

659

660

661

662

663

664

665

666

667

668

669

670

671

672

673

674

675

Holzschuh A, Dormann CF, Tscharntke T, and Steffan-Dewenter I. 2011. Expansion of massflowering crops leads to transient pollinator dilution and reduced wild plant pollination. Proceedings of the Royal Society B-Biological Sciences 278:3444-3451.

Jakobsen HB, and Kritjánsson K. 1994. Influence of Temperature and Floret Age on Nectar Secretion in Trifolium repens L. Annals of Botany 74:327-334.

Krupke CH, Hunt GJ, Eitzer BD, Andino G, and Given K. 2012. Multiple Routes of Pesticide Exposure for Honey Bees Living Near Agricultural Fields. PloS one 7.

PDOK. 2015. TOP10NL. Available at https://www.pdok.nl/nl/producten/pdok-downloads/basisregistratie-topografie/topnl/topnl-actueel/top 10nl.

Peat J, Tucker J, and Goulson D. 2005. Does intraspecific size variation in bumblebees allow colonies to efficiently exploit different flowers? Ecological Entomology 30:176-181.

Pierre J, Mesquida J, Marilleau R, Pham-Delègue MH, and Renard M. 1999. Nectar secretion in winter oilseed rape, Brassica napus - quantitative and qualitative variability among 71 genotypes. Plant Breeding 118:471-476.

Potts SG, Biesmeijer JC, Kremen C, Neumann P, Schweiger O, and Kunin WE. 2010. Global pollinator declines: trends, impacts and drivers. Trends in Ecology \& Evolution 25:345353.

Requier F, Odoux J-F, Tamic T, Moreau N, Henry M, Decourtye A, and Bretagnolle V. 2015. Honey bee diet in intensive farmland habitats reveals an unexpectedly high flower richness and a major role of weeds. Ecological Applications 25:881-890.

Ricketts TH, Regetz J, Steffan-Dewenter I, Cunningham SA, Kremen C, Bogdanski A, GemmillHerren B, Greenleaf SS, Klein AM, Mayfield MM, Morandin LA, Ochieng A, and Viana BF. 2008. Landscape effects on crop pollination services: are there general patterns? Ecology Letters 11:499-515.

Riedinger V, Renner M, Rundlof M, Steffan-Dewenter I, and Holzschuh A. 2014. Early massflowering crops mitigate pollinator dilution in late-flowering crops. Landscape Ecology 29:425-435.

Rortais A, Arnold G, Halm MP, and Touffet-Briens F. 2005. Modes of honeybees exposure to systemic insecticides: estimated amounts of contaminated pollen and nectar consumed by different categories of bees. Apidologie 36:71-83. 
676 Schmickl T, and Crailsheim K. 2007. HoPoMo: A model of honeybee intracolonial population 677 dynamics and resource management. Ecological Modelling 204:219-245.

678 Schmid-Hempel P, Kacelnik A, and Houston AI. 1985. Honeybees Maximize Efficiency by Not 679 Filling Their Crop. Behavioral Ecology and Sociobiology 17:61-66.

680 Schneider SS. 1989. SPATIAL FORAGING PATTERNS OF THE AFRICAN HONEY BEE, 681 APIS-MELLIFERA-SCUTELLATA. Journal of Insect Behavior 2:505-521.

Seeley T. 1995. The wisdom of the hive. Cambridge, MA: Harvard University Press.

Seeley TD. 1985. Honeybee Ecology: A Study of Adaptation in Social Life: Princeton University Press.

Seeley TD. 1986. SOCIAL FORAGING BY HONEYBEES - HOW COLONIES ALLOCATE FORAGERS AMONG PATCHES OF FLOWERS. Behavioral Ecology and Sociobiology 19:343-354.

Seeley TD. 1994. HONEY-BEE FORAGERS AS SENSORY UNITS OF THEIR COLONIES. Behavioral Ecology and Sociobiology 34:51-62.

Seeley TD, Camazine S, and Sneyd J. 1991. COLLECTIVE DECISION-MAKING IN HONEYBEES - HOW COLONIES CHOOSE AMONG NECTAR SOURCES. Behavioral Ecology and Sociobiology 28:277-290.

Seeley TD, and Visscher PK. 1988. ASSESSING THE BENEFITS OF COOPERATION IN HONEYBEE FORAGING - SEARCH COSTS, FORAGE QUALITY, AND COMPETITIVE ABILITY. Behavioral Ecology and Sociobiology 22:229-237.

Sponsler DB, and Johnson RM. 2015. Honey bee success predicted by landscape composition in Ohio, USA. Peerj 3:e838.

Stephens DW, and Krebs JR. 1987. Foraging Theory. Princeton, NJ: Princeton University Press.

Vanbergen AJ, Baude M, Biesmeijer JC, Britton NF, Brown MJF, Brown M, Bryden J, Budge GE, Bull JC, Carvell C, Challinor AJ, Connolly CN, Evans DJ, Feil EJ, Garratt MP, Greco MK, Heard MS, Jansen VAA, Keeling MJ, Kunis WE, Marris GC, Memmott J, Murray JT, Nicolson SW, Osborne JL, Paxton RJ, Pirk CWW, Polce C, Potts SG, Priest NK, Raine NE, Roberts S, Ryabov EV, Shafir S, Shirley MDF, Simpson SJ, Stevenson PC, Stone GN, Termansen M, Wright GA, and Insect Pollinators I. 2013. Threats to an ecosystem service: pressures on pollinators. Frontiers in Ecology and the Environment $11: 251-259$. 
707 Visscher PK, and Seeley TD. 1982. FORAGING STRATEGY OF HONEYBEE COLONIES IN A TEMPERATE DECIDUOUS FOREST. Ecology 63:1790-1801.

Winfree R, Aguilar R, Vazquez DP, LeBuhn G, and Aizen MA. 2009. A meta-analysis of bees' responses to anthropogenic disturbance. Ecology 90:2068-2076.

Winston ML. 1987. The biology of the honey bee: Harvard University Press, Cambridge, Massachusetts.

Wratten SD, Gillespie M, Decourtye A, Mader E, and Desneux N. 2012. Pollinator habitat enhancement: Benefits to other ecosystem services. Agriculture Ecosystems \& Environment 159:112-122. 


\section{Table 1 (on next page)}

Fixed (energetic) coefficients for honeybee and nectar 
1 Table 1.

\begin{tabular}{|l|l|l|l|l|}
\hline Coefficient & Symbol & dimension & Value & source \\
\hline Maximum foraging distance & $D_{\max }$ & $\mathrm{m}$ & $2000^{1}$ & \\
\hline Flying speed & $v$ & $\mathrm{~m} \mathrm{~s}^{-1}$ & $4.17^{2}$ & $\begin{array}{l}\text { (de Vries \& Biesmeijer } \\
1998)\end{array}$ \\
\hline Flight cost (unloaded) & $e_{U}$ & $\mathrm{~J} \mathrm{~s}^{-1}$ & $0.037^{3}$ & (Seeley 1986) \\
\hline Flight cost (loaded) & $e_{L}$ & $\mathrm{~J} \mathrm{~s}^{-1}$ & 0.075 & (Seeley 1986) \\
\hline Capacity (maximum load) & $\gamma$ & $\mathrm{mg}$ & 32.5 & (Winston 1987) \\
\hline Energetic value sugar & $e_{S U G A R}$ & $\mathrm{~J} \mathrm{mg}^{-1}$ sugar & 17.2 & (Seeley 1985) \\
\hline $\begin{array}{l}\text { Time unloading nectar and } \\
\text { dancing }\end{array}$ & $t_{U D}$ & $\mathrm{~s}$ & $300^{4}$ & (Seeley et al. 1991) \\
\hline
\end{tabular}

2

$3 \quad{ }^{1}$ Specific for studied scenarios. Considerably longer distances have been reported, e.g. max. 13.5 $4 \mathrm{~km}, 95 \%$ within $6 \mathrm{~km}$, mean $2.3 \mathrm{~km}$ (Beekman \& Ratnieks 2000). ${ }^{2}$ Other reported value $6.95 \mathrm{~m}$ $5 \mathrm{~s}^{-1}$ (Seeley 1995). ${ }^{3}$ Other reported value $0.0334 \mathrm{~J} \mathrm{~s}^{-1}$ (Schmid-Hempel et al. 1985 and references

6 therein). ${ }^{4}$ Goodwin et al. (2011) observed longer times in the hive between foraging (11.13

7 minutes). Range 150-300 in the model of Seeley et al. (1991). 


\section{Table 2(on next page)}

\section{Resource-specificcoefficients}

Coefficient values for the two resources considered in the study, oil-seed rape and clover 
1 Table 2.

\begin{tabular}{|l|l|l|r|r|r|}
\hline \multicolumn{2}{|l|}{ Crop specific parameters } & & Oil-seed rape & Off-field (clover) \\
\hline & $g$ & nectar per flower & $\mathrm{mg}$ & $1.071^{1}$ & $0.373^{2}$ \\
\hline & $F_{0}$ & open flowers density & $\mathrm{m}^{-2}$ & $264^{3}$ & $2808^{4}$ \\
\hline & & share of sugar in nectar & $\mathrm{g} \mathrm{g}^{-1}$ & $0.47^{5}$ & $0.74^{6}$ \\
\hline & $e_{R}$ & energetic content nectar & $\mathrm{J} \mathrm{mg}^{-1}$ & $8.084^{7}$ & $12.728^{8}$ \\
\hline & $a$ & attack rate & $\mathrm{m}^{2} \mathrm{~s}^{-1}$ & $0.00147^{9}$ & $0.00020^{10}$ \\
\hline & $h$ & handling time per flower & $\mathrm{s}$ & $4.1^{11}$ & $1.22^{12}$ \\
\hline
\end{tabular}

2

$3{ }^{1}$ Range $0.7-6 \mu \mathrm{l}$ (average $2.0 \mu \mathrm{l}$ ) (Pierre et al. 1999 ), approximately $0.7+0.7 * 0.53=1.071$ to

$46+6 * 0.53=9.18 \mathrm{mg}$

$5 \quad{ }^{2}$ Range $0.143-0.351\left(10^{\circ} \mathrm{C}\right)$ and $0.112-0.373\left(18^{\circ} \mathrm{C}\right)($ Jakobsen \& Kritjánsson 1994$)$

$6{ }^{3}$ Own measurements: (June 2014: 1182; September 2015: 264)

$7{ }^{4}$ (Goodwin et al. 2011) for clover field, defined as density of open florets ( $=15.6$ open florets per

8 flower x 180 flowers $\mathrm{m}^{-2}$ ). (Burdon 1983): 1500-3000 open flowers, derived from 20-40 florets

9 per flower-head (average 30), assuming $50-100$ flower-heads per $\mathrm{m}^{2}$

10 5Range 0.47-0.59 compiled from: Günter Pritsch, 2007, Bienenweide, Franckh-Kosmos verlag-

11 Gmbh; S.E. McGrego, 1976, Insect Pollination of Cultivated Crop Plants, Agriculture Handbook

12 No. 496, Agricultural Research Service US Dep. Of Agriculture; Anna Maurizio \& Ina Grafl,

13 1969, Das Trachtpflanzenbuch, Ehrenwirth Verlag, München

14 'Jakobsen \& Kritjánsson (1994)

$15 \quad{ }^{7,8}$ From (share of sugar) $* e_{S U G A R}$

$16{ }^{9,10}$ See SI.

$17{ }^{11}$ Free \& Nuttall (1968)

$18{ }^{12}$ Peat et al. (2005) for small bumble bee 


\section{Figure 1}

Map of the case-study area

Oil-seed rape fields (ochre), alternative crop fields (lilac) and off-field resource patches (green) as used in the scenario simulations.

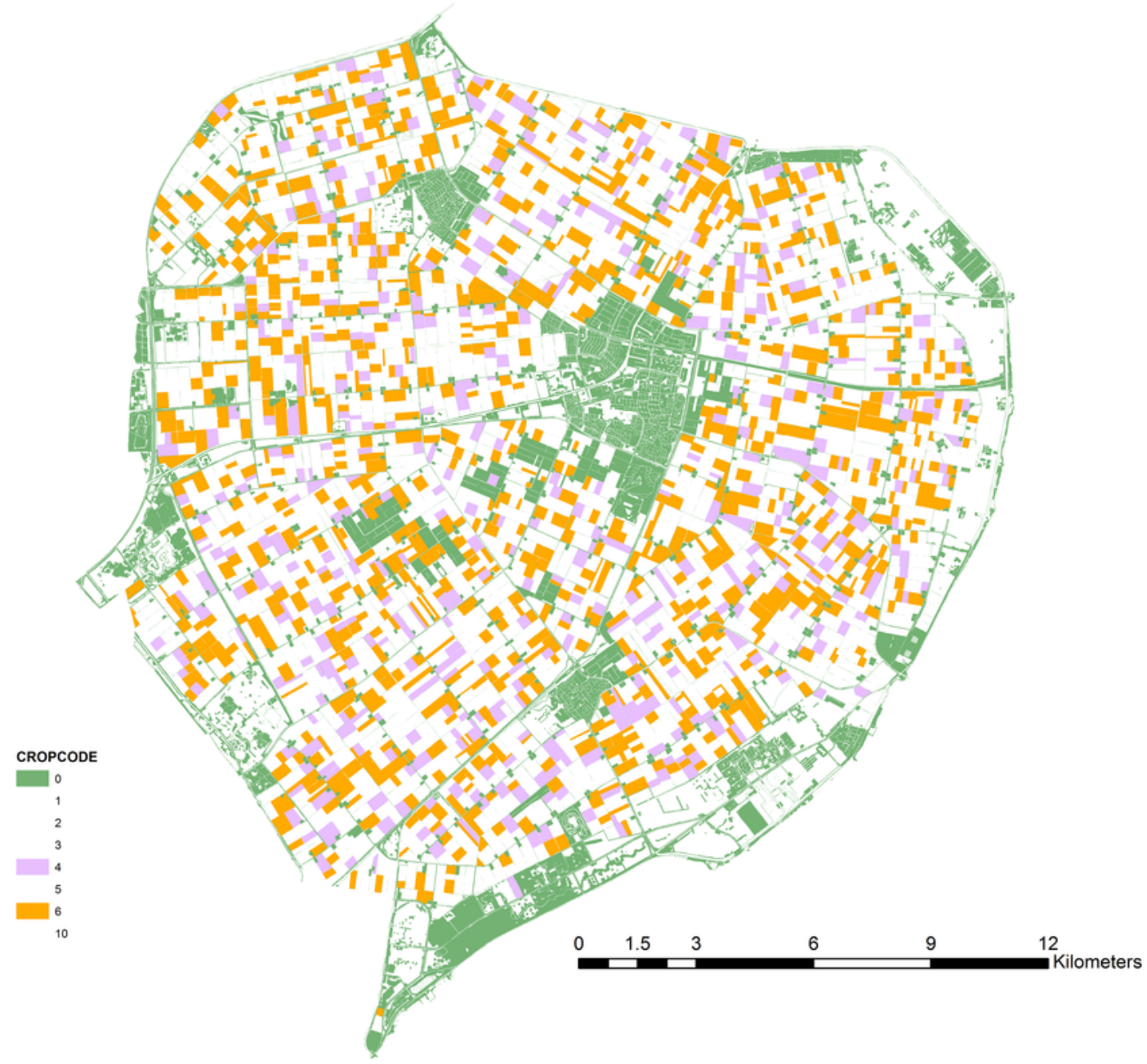




\section{Figure 2 (on next page)}

Choice of resources depends on net energetic efficiency

The choice between resources at equal distance depends on their value for EI/EE (Eq. 22b). This ratio increases asymptotically with nectar acquisition rate gaF to the limit value $1 / c \times e_{R} / e_{F}$. With larger $e_{R}$ , e.g., for clover compared to OSR, EI/EE will level off at a higher value. When flower density is much higher, the resulting value of EI/EE may still be larger for the resource with the lower $e_{R}$. Curves refer to resources at $200 \mathrm{~m}$ and $1 \mathrm{~km}$. Values for clover and OSR as specified in table 2 and used in the scenarios are indicated. 


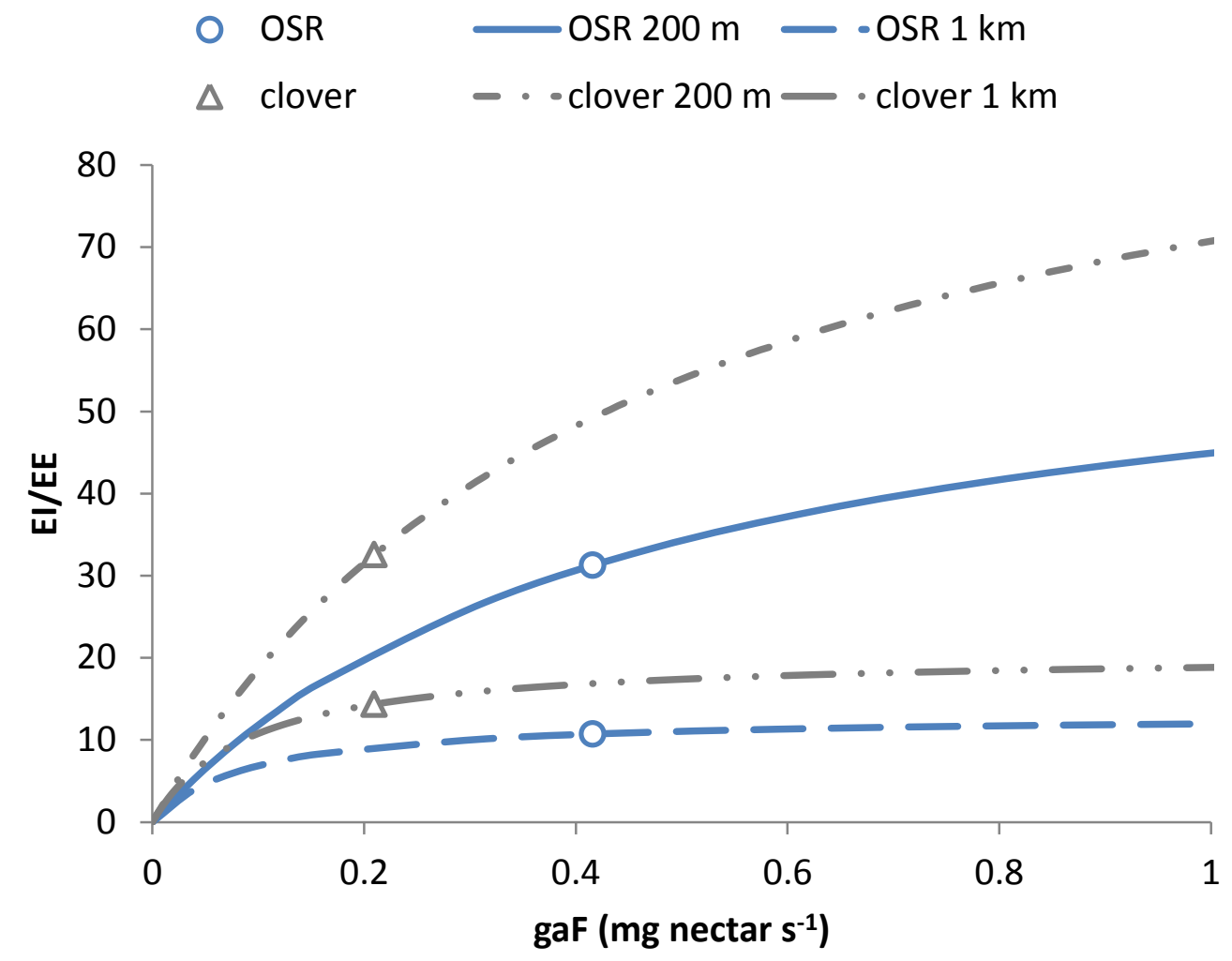

Figure 2 


\section{Figure 3 (on next page)}

Attractive flower strips and off-field habitats may dilute exposure

Flower strips can lead to high exposure reduction, when present in large numbers (a) For the RL-model, the width of the strips has no impact on this dilution; for 2, 5 and $10 \mathrm{~m}$ wide strips, the graphs are very similar (not shown). Off-field habitats can have similar impact $(b, c)$ as flower strips. When quality is low (d) few offfield habitat patches are selected resulting in little dilution. Note that flowers strips are identical to medium quality off-field habitat patches. 
(a) "Flower Strips" $1 \mathrm{~m}$
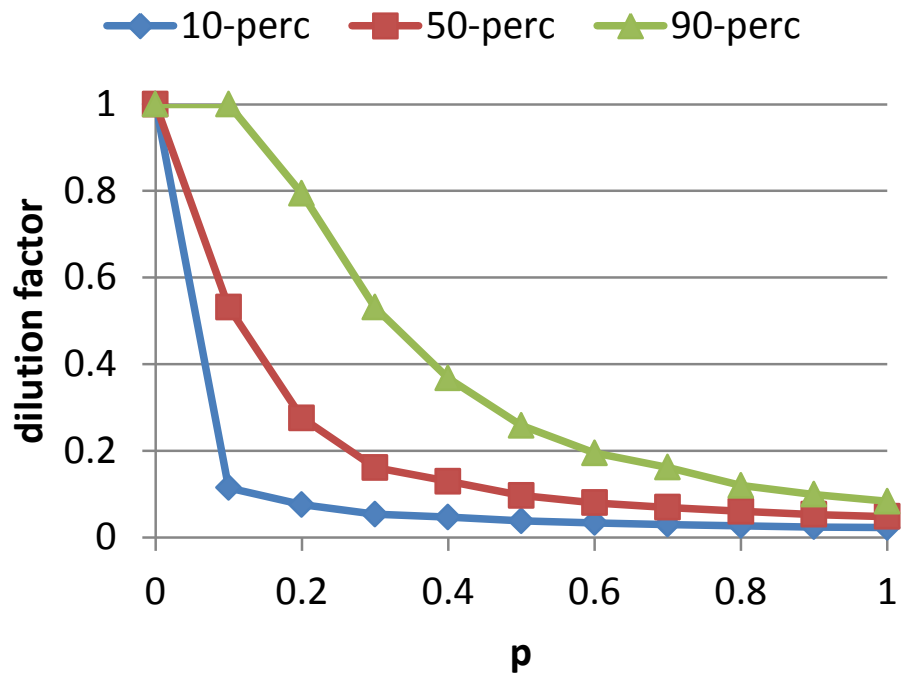

(c) "Off-field Habitats" medium quality
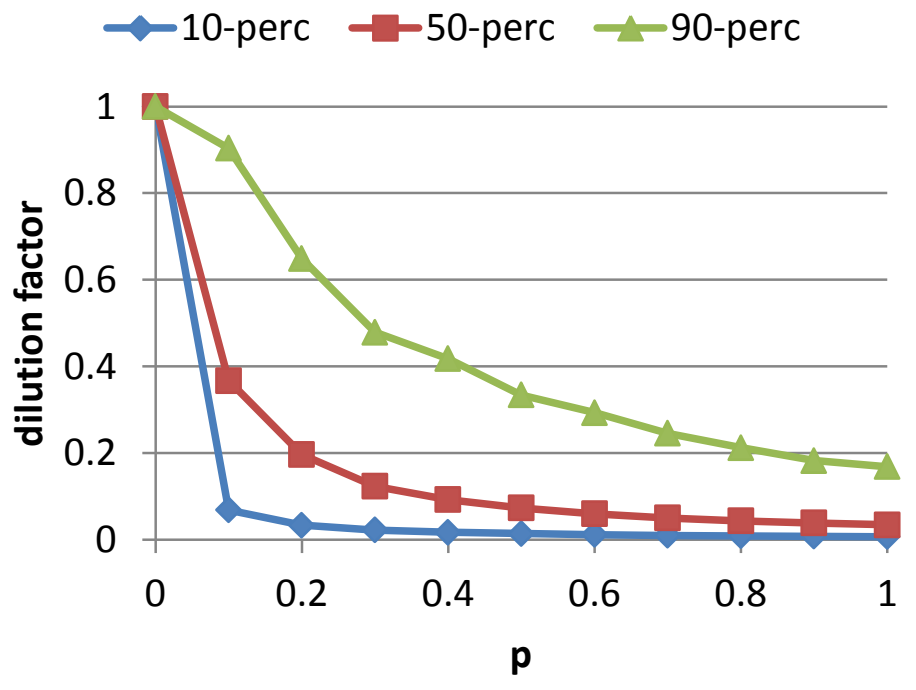

(b) "Off-field Habitats" high quality
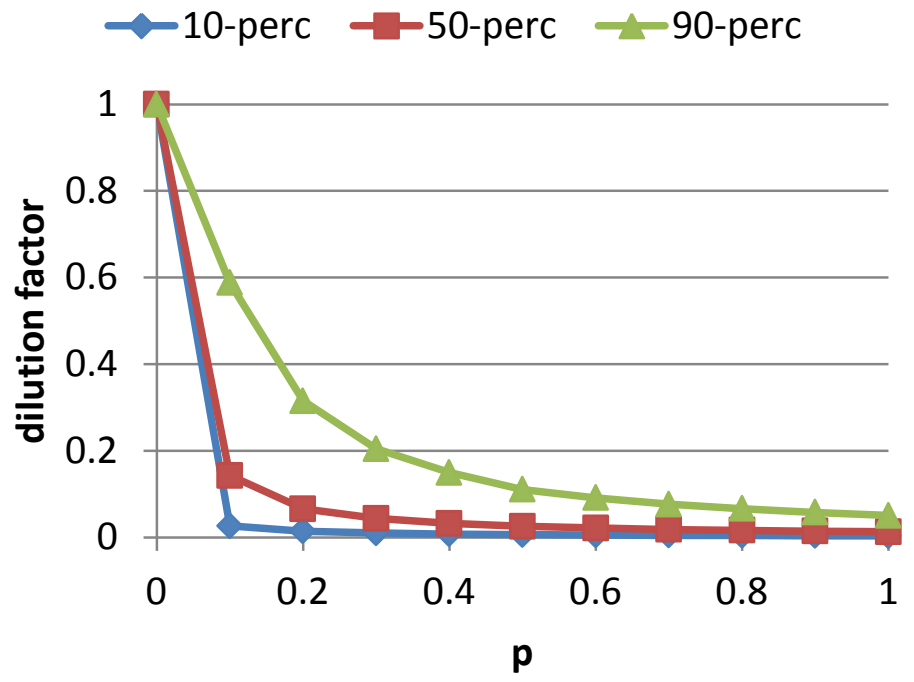

(d) "Off-field Habitats" low quality

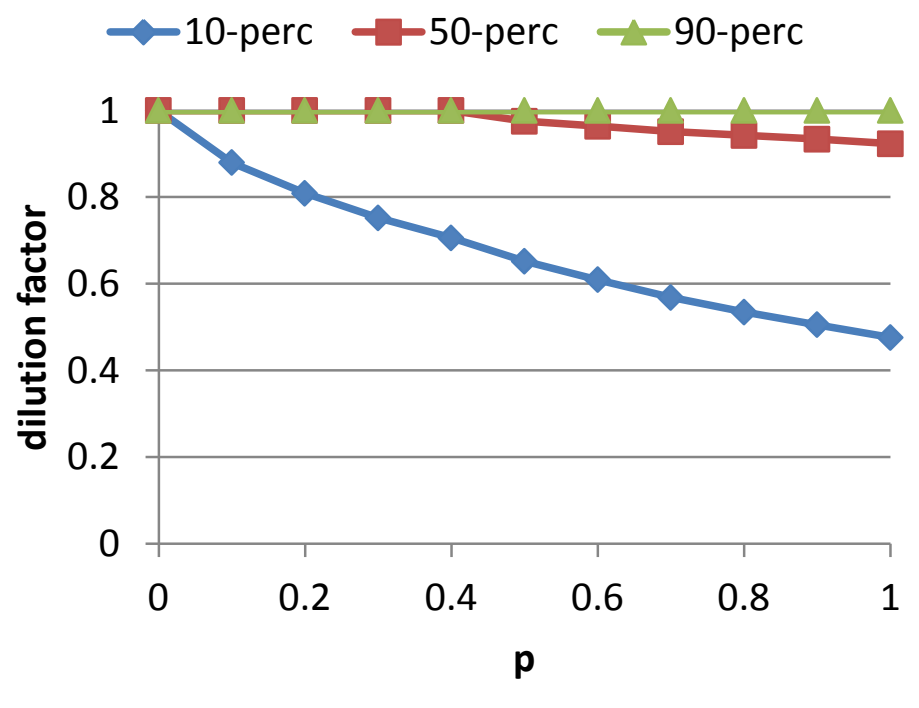

Figure 3 


\section{Figure 4 (on next page)}

The number of daily exploited patches depends on the foraging patch selection model

With the SO-model an increase in the number of exploited patches indicates that depletion of the optimal patch plays a role. This may occur for narrow flower strips (a) and for off-field habitats that are mostly small-sized (c), and is determined by patch size not by its 'quality'. For the RL-model the number of exploited patches is linearly related to their numerousness. Depletion is unlikely and patch size has no impact on patch selection (b). Instead patch 'quality' is important (d) as patches need to have a sufficiently high NEE to be considered and promoted for in the recruitment process. 
(a) SO-model "Flower Strips" 1,2 and $5 \mathrm{~m}$

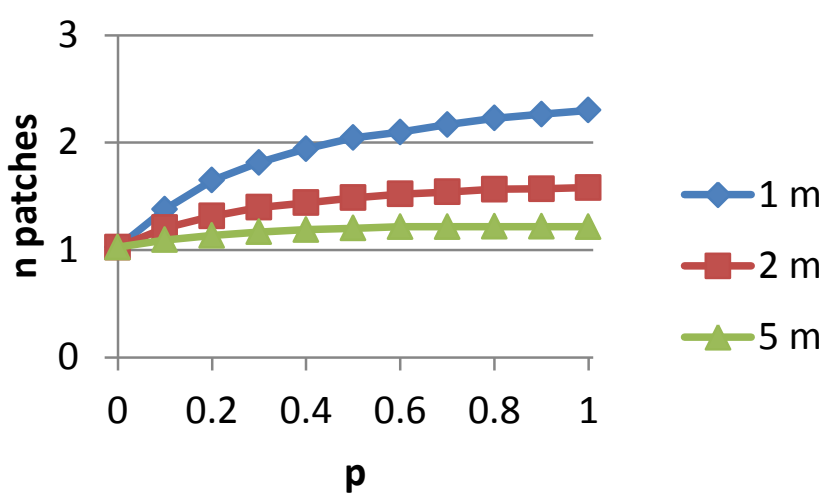

(c) SO-model "Off-field Habitats" high, medium and low quality

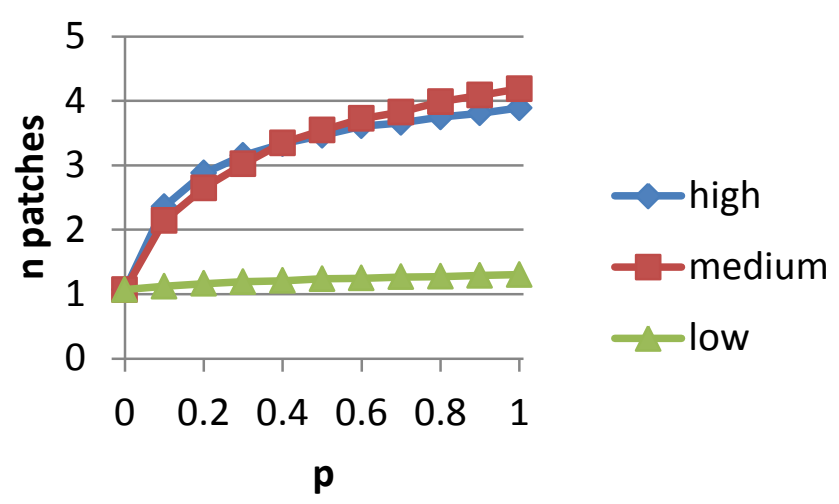

(b) RL-model "Flower Strips" 1, 2 and $5 \mathrm{~m}$

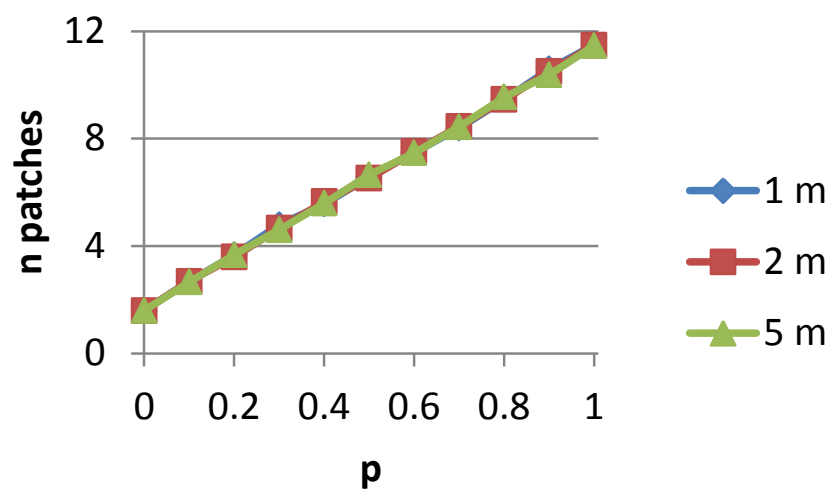

(d) RL-model "Off-field Habitats" high, medium and low quality

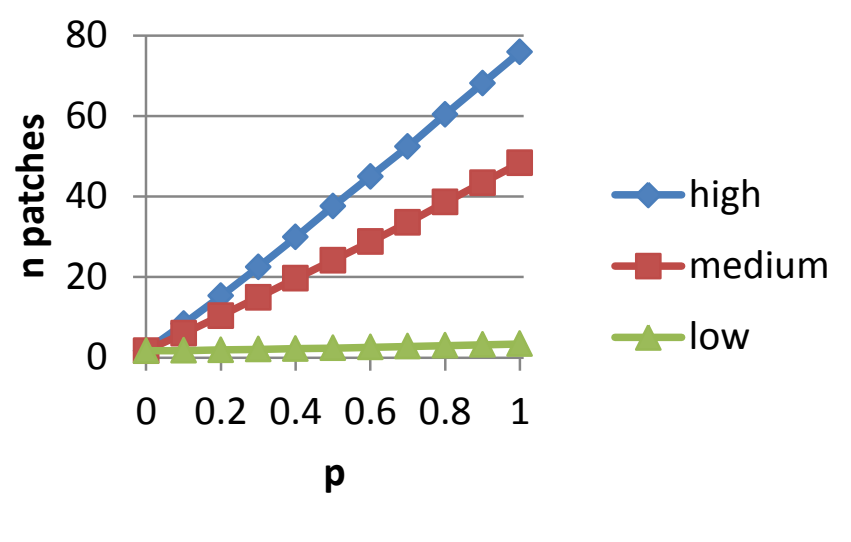

Figure 4 


\section{Figure 5 (on next page)}

On an area-base flower strips are more effective than off-field patches

To obtain the same degree of dilution, much more area is required of managed off-field habitat patches (b), compared to flower strips (a) with identical properties. This is related to landscape characteristics and a consequence of off-field patches being in general further away from target crop field and hive location. 
(a) "Flower Strips" $1 \mathrm{~m}$

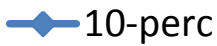

50-perc

$+90-$ perc

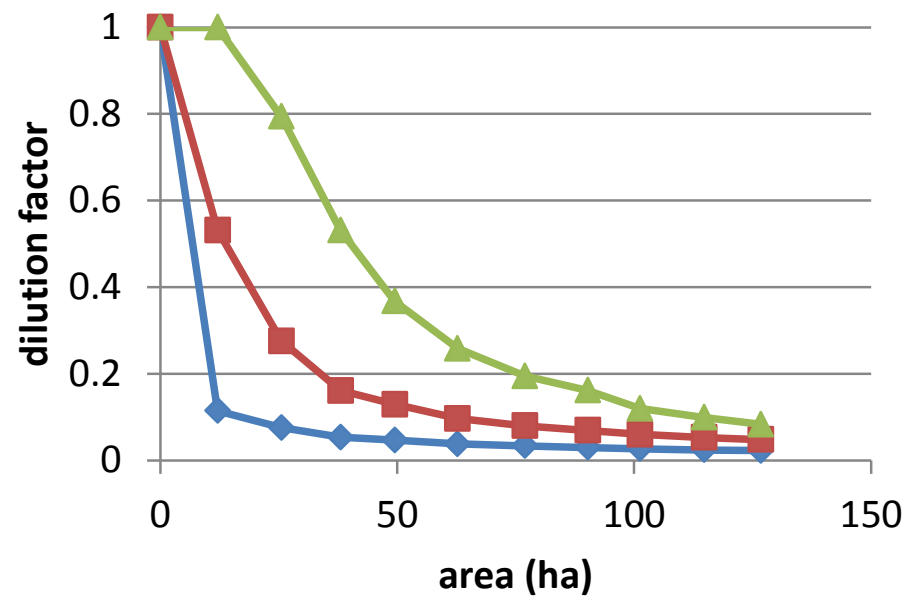

(b) "Off-field Habitats" medium quality

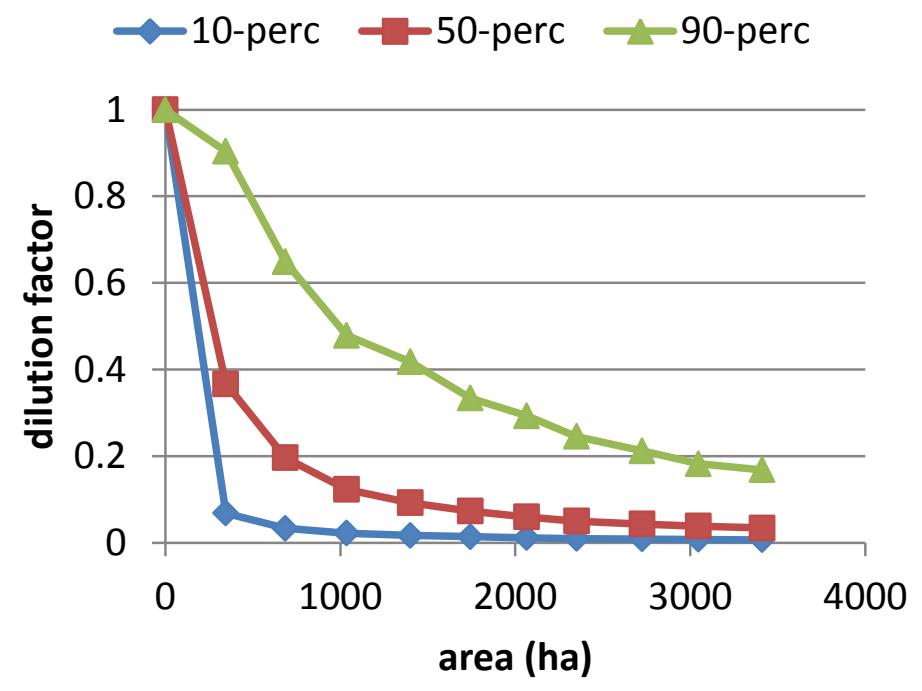

Figure 5 